TITLE:

\title{
External forcing and feedback control of nonlinear dissipative waves
}

$\operatorname{AUTHOR}(S):$

Tokuda, H; Zykov, VS; Ohta, T

\section{CITATION:}

Tokuda, $H$...[et al]. External forcing and feedback control of nonlinear dissipative waves. Physical Review E 2007, 75(6): 066203.

ISSUE DATE:

2007-06

URL:

http://hdl.handle.net/2433/50027

RIGHT:

Copyright 2007 American Physical Society 
PHYSICAL REVIEW E 75, 066203 (2007)

\title{
External forcing and feedback control of nonlinear dissipative waves
}

\author{
Hidekazu Tokuda, ${ }^{1, *}$ Vladimir S. Zykov, ${ }^{2, \dagger}$ and Takao Ohta ${ }^{1, \$}$ \\ ${ }^{1}$ Department of Physics, Kyoto University, Kyoto, 606-8502, Japan \\ ${ }^{2}$ Institut für Theoretische Physik, Technische Universität Berlin, Hardenbergstrasse 36, D-10623 Berlin, Germany
}

(Received 26 December 2006; published 7 June 2007)

\begin{abstract}
The dynamics of traveling waves in a nonlinear dissipative system are studied analytically and numerically. Spatiotemporal forcing and feedback forcing are applied to the traveling waves in a phase-separated system undergoing chemical reactions. The stability of the traveling waves and interesting, unexpected behavior, including the reversal of the propagation direction are analyzed in one dimension. The phase dynamical approach is applied to gain a theoretical understanding of the dynamics.
\end{abstract}

DOI: 10.1103/PhysRevE.75.066203

PACS number(s): 82.40.Ck, 47.54.-r

\section{INTRODUCTION}

The self-organized formation of spatiotemporal patterns constitutes a general class of phenomena exhibited by nonlinear, nonequilibrium systems [1]. Such phenomena have been the subject of extensive theoretical and experimental research in physics, biology, and chemistry [2-6]. One important topic in these investigations is the elaboration of mechanisms and methods capable of controlling the spontaneous pattern formation processes. Such control would allow for simplification of the study of complex systems, e.g., by stabilization of some unstable dynamical regimes, and has potential applications in a wide range of fields, from laser and plasma physics, hydrodynamics and material sciences to cardiology [7-15].

The external forcing applied to a controlled system can be applied in a predetermined manner or can be generated as a feedback signal. Numerous studies of "lumped" systems without spatial degrees of freedom have demonstrated that the dynamics of oscillating systems subjected to pure periodic forcing and those subjected to a feedback-mediated control possess many common features $[16,17]$. However, the feedback control makes possible certain ways of controlling the behavior of dynamics systems, and through its application, one can create dynamical regimes that cannot be realized with pure periodic forcing $[16,17]$.

The control of spatially extended systems is potentially very important for many applications, but it is much more complicated than the control of lumped systems, because of the existence of many degrees of freedom. While in many of the cases studied to this time, spatially uniform forcing has been employed, spatiotemporal control has attracted considerable attention in the last decade [18-23]. Such spatially nonuniform control is, obviously, a specific feature of distributed systems and represents an important problem in the study of modern nonlinear dynamics.

An important example of self-organized structures is spatiotemporal oscillations in distributed systems near a Hopf or a Turing-Hopf bifurcation. In particular, it was recently

\footnotetext{
*Electronic address: tokuda@ton.scphys.kyoto-u.ac.jp

${ }^{\dagger}$ Electronic address: zykov@ raphael.physik.TU-Berlin.DE

"Electronic address: takao@scphys.kyoto-u.ac.jp
}

shown that in phase separation mixtures undergoing chemical reactions under certain conditions self-sustained traveling waves appear [24-26]. It was demonstrated that such waves can be effectively controlled through the application of spatiotemporal forcing [27,28]. Under such control, the traveling waves can be entrained by external forcing, as in the case of nonlinear lumped oscillators subject to periodic perturbation.

The purpose of this paper is to theoretically investigate the dynamics of traveling waves under feedback-mediated spatiotemporal control. For comparison, some new results of the dynamics under spatiotemporal external forcing are also presented. It is shown that, as in the case of "lumped" oscillators, feedback control will reveal spatiotemporal dynamics of traveling waves. In addition, we develop a theory in terms of the phase variables for spatiotemporal control of traveling waves in distributed nonequilibrium systems.

The organization of this paper is as follows. In the next section, we briefly review our model system. This system undergoes a Hopf bifurcation at a finite wave number, and beyond this point, propagating wave is self-organized. In Sec. III, we report numerical results obtained from the simulations of the entrainment of this wave to external forcing. Then, in Sec. IV, we discuss the numerical simulations of feedback forcing. There, we demonstrate the existence of an interesting phenomenon of propagation reversal under feedback forcing. The phase dynamics approach is formulated both for external forcing, in Sec. V, and for feedback forcing in Sec. VI. We find that all of the results obtained in the simulations are quantitatively consistent with the results derived from the phase equations. The summary and discussion are given in Sec. VII.

\section{MODEL EQUATION AND TRAVELING WAVES}

Throughout this paper, we employ model equations for traveling waves in a phase separating system undergoing chemical reactions [24-26]. We consider a mixture of three chemical components, $A, B$ and $C$, and assume that the $A$ and $B$ species tend to segregate and that there is a cyclic chemical reaction represented by

$$
A \stackrel{\gamma_{1}}{\rightarrow} B \stackrel{\gamma_{2}}{\rightarrow} C \stackrel{\gamma_{3}}{\longrightarrow} A,
$$

with the reaction rates $\gamma_{1}, \gamma_{2}$, and $\gamma_{3}$. 
Let us assume that molecules $A, B$, and $C$ are adsorbed on a flat substrate with the local concentrations, $\psi_{A}, \psi_{B}$, and $\psi_{C}$, respectively. The other chemical species involved in the chemical reactions are assumed to exist abundantly in the gas phase above the substrate, and the products are also assumed to dissolve quickly into the gas phase. Each lattice site of the substrate is occupied by one and only one molecule $A, B$, or $C$. Any pair of molecules $A$ and $B$ that are nearest neighbors exchange their positions randomly with a certain probability, but $C$ molecules do not participate in such exchanges. In this way, the condition $\psi_{A}+\psi_{B}+\psi_{C}=1$ is satisfied in the continuum limit, while diffusion is exhibited by $A$ and $B$ molecules but not $C$ molecules. Then the time-evolution equations for the local concentrations $\psi=\psi_{A}-\psi_{B}$ and $\phi=\psi_{A}+\psi_{B}$ are given by [24]

$$
\begin{gathered}
\frac{\partial \psi}{\partial t}=\nabla^{2} \frac{\delta F}{\delta \psi}+f(\psi, \phi), \\
\frac{\partial \phi}{\partial t}=g(\psi, \phi) .
\end{gathered}
$$

Here the free energy functional $F$ is assumed to be of the Cahn-Hilliard type for phase separation,

$$
F=\int d \mathbf{r}\left(\frac{D}{2}(\nabla \psi)^{2}-\frac{\tau}{2} \psi^{2}+\frac{1}{4} \psi^{4}\right),
$$

where $D$ and $\tau$ are positive constants.

The terms $f$ and $g$ in Eqs. (2) and (3) arise from the chemical reaction (1) and are given by

$$
\begin{gathered}
f(\psi, \phi)=-\left(\gamma_{1}+\frac{\gamma_{2}}{2}\right) \psi-\left(\gamma_{1}-\frac{\gamma_{2}}{2}+\gamma_{3}\right) \phi+\gamma_{3}, \\
g(\psi, \phi)=\frac{\gamma_{2}}{2} \psi-\left(\frac{\gamma_{2}}{2}+\gamma_{3}\right) \phi+\gamma_{3} .
\end{gathered}
$$

As mentioned above, there is no diffusion term in Eq. (3). We have verified that the inclusion of such a term representing the diffusion of $\phi$ does not alter significantly the dynamics described below [25].

Setting $f=g=0$, the uniform stationary solution of Eqs. (2) and (3) is readily obtained as

$$
\begin{aligned}
& \psi_{0}=\frac{\gamma_{3}\left(\gamma_{2}-\gamma_{1}\right)}{\gamma_{1} \gamma_{2}+\gamma_{2} \gamma_{3}+\gamma_{3} \gamma_{1}}, \\
& \phi_{0}=\frac{\gamma_{3}\left(\gamma_{2}+\gamma_{1}\right)}{\gamma_{1} \gamma_{2}+\gamma_{2} \gamma_{3}+\gamma_{3} \gamma_{1}} .
\end{aligned}
$$

Applying a linear stability analysis of the steady uniform solution (7) and (8), we obtain the bifurcation diagram displayed in Fig. 1 [25], where the parameters are set as $D=1$, $\gamma_{1}=0.3$, and $\gamma_{3}=0.05$, and the remaining two parameters, $\tau$ and $\gamma_{2}$, are varied. A Hopf bifurcation at a finite wave number appears at the solid curve and a Turing-type bifurcation occurs at the dotted curve. A motionless periodic pattern appears in the region indicated by the symbols $X$, whereas a propagating wave pattern appears in the region indicated by

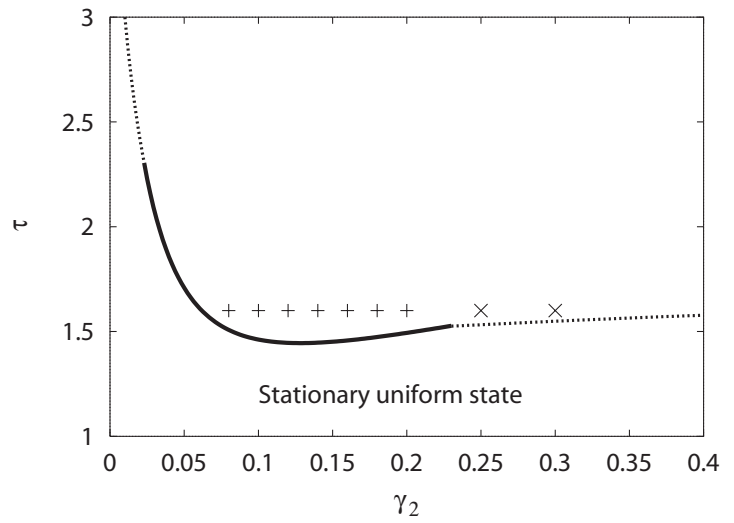

FIG. 1. Bifurcation diagram for the uniform stationary solution with $D=1, \gamma_{1}=0.3$, and $\gamma_{3}=0.05$. The solid curve and dotted curve represents the Hopf bifurcation line and the Turing-type bifurcation line, respectively. A traveling wave appears at the area indicated by the symbols + , whereas a motionless pattern appears at the area indicated by the symbols $x$.

the symbols + . Figure 2 plots the spatial variation of a propagating wave obtained numerically.

Just at the Hopf bifurcation line, the local concentrations are given by

$$
\begin{gathered}
\psi(x, t)=\psi_{0}+\Psi \cos \left( \pm q_{c} x-\omega_{c} t\right), \\
\phi(x, t)=\phi_{0}+\Phi \cos \left( \pm q_{c} x-\omega_{c} t+\theta\right),
\end{gathered}
$$

where the amplitudes $\Psi$ and $\Phi$ cannot be determined within the linear theory. The phase difference is given by

$$
\theta=\tan ^{-1}\left(\frac{2 \omega_{c}}{\gamma_{2}+2 \gamma_{3}}\right),
$$

which yields $\theta \approx 0.494$ for $\gamma_{2}=0.16$. This value is very close to that obtained from Fig. 2 numerically. For $\tau>3 \psi_{0}^{2}$, the critical wave number $q_{c}$, the bifurcation point $\tau_{c}$, and the critical frequency $\omega_{c}$ are given by

$$
q_{c}=\left(\frac{\tau-3 \psi_{0}^{2}}{2}\right)^{1 / 2},
$$

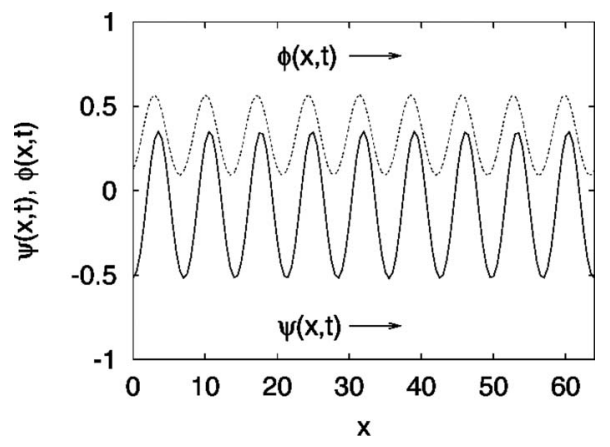

FIG. 2. Spatial profiles of $\psi(x, t)$ (solid curve) and $\phi(x, t)$ (dashed curve) for $D=1.0, \tau=1.6, \gamma_{1}=0.3, \gamma_{2}=0.16$, and $\gamma_{3}=0.05$. Both $\psi(x, t)$ and $\phi(x, t)$ are propagating to the right at the same speed. 


$$
\begin{gathered}
\tau_{c}=3 \psi_{0}^{2}+2\left(\gamma_{1}+\gamma_{2}+\gamma_{3}\right)^{1 / 2}, \\
\omega_{c}=\left(\frac{\gamma_{1} \gamma_{2}-\gamma_{2} \gamma_{3}-\gamma_{2}^{2}}{2}-\gamma_{3}^{2}\right)^{1 / 2} .
\end{gathered}
$$

The numerical values of these critical quantities are $\tau_{c}$ $\approx 1.46, q_{c} \approx 0.9$, and $\omega_{c} \approx 0.07$ for $D=1.0, \tau=1.6, \gamma_{1}=0.3$, $\gamma_{2}=0.16$, and $\gamma_{3}=0.05$.

Before closing this section, we briefly explain the reason that we call the bifurcation indicated by the dotted curve in Fig. 1 a Turing-type bifurcation [25]. The usual Turing instability [29] is a phenomenon exhibited by a two-component reaction diffusion system which takes the form

$$
\begin{aligned}
& \frac{\partial u}{\partial t}=D_{u} \nabla^{2} u+r_{u}(u, v), \\
& \frac{\partial v}{\partial t}=D_{v} \nabla^{2} v+r_{v}(u, v),
\end{aligned}
$$

where $D_{u}$ and $D_{v}$ are the diffusion constants, and $r_{u}$ and $r_{v}$ represent the reactions. Turing showed that if the ratio of the diffusion constants and the nature of the nonlinearity possessed by $r_{u}$ and $r_{v}$ satisfy certain conditions, the uniform time-independent solution of Eqs. (15) and (16) is unstable, and the nonuniform periodic solution can be stable in equilibrium. Now, note that Eq. (2) with (4) contains the fourth spatial derivative of $\psi$. This term together with the second derivative term is the origin of the periodic solution in the present system. This is different from the mechanism proposed by Turing and hence we call the bifurcation not a Turing bifurcation but a Turing-type bifurcation. It should also be mentioned that there is no Hopf bifurcation at a finite wave number in a two-component reaction diffusion system like Eqs. (15) and (16).

\section{EXTERNAL FORCING}

The stability and modulation of traveling waves under external forcing has been studied in one dimension $[27,28]$. Spatiotemporal forcing is added to Eqs. (2) and (3) as

$$
\frac{\partial \psi}{\partial t}=\nabla^{2}\left(-\nabla^{2} \psi-\tau \psi+\psi^{3}\right)+a_{1} \psi+a_{2} \phi+a_{3}+\Gamma(x, t),
$$

$$
\frac{\partial \phi}{\partial t}=b_{1} \psi+b_{2} \phi+b_{3}+\Gamma(x, t)
$$

where

$$
\Gamma(x, t)=\epsilon \cos \left(q_{f} x-\Omega t\right),
$$

with the strength of the external forcing $\epsilon$, the wave number $q_{f}$, and the external frequency $\Omega$. The constants in Eqs. (17) and (18) are given by

$$
a_{1}=-\left(\gamma_{1}+\frac{\gamma_{2}}{2}\right)
$$

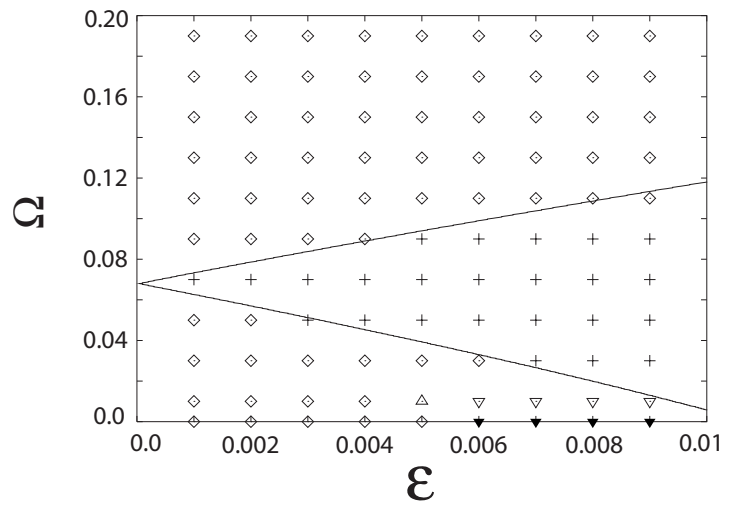

FIG. 3. Phase diagram for $\gamma_{2}=0.16, \tau=1.6$, and $q_{f}=q_{c}$. The solid line is the theoretically obtained stability limit of the unmodulated traveling wave possessing the same velocity as the external force. The meanings of the other symbols are explained in Ref. [27], and they are unimportant in the present study.

$$
\begin{gathered}
a_{2}=-\left(\gamma_{1}-\frac{\gamma_{2}}{2}+\gamma_{3}\right), \\
a_{3}=\gamma_{3}, \\
b_{1}=\frac{\gamma_{2}}{2}, \\
b_{2}=-\left(\frac{\gamma_{2}}{2}+\gamma_{3}\right), \\
b_{3}=\gamma_{3} .
\end{gathered}
$$

In the present study, we restrict our inquiry to the case that the spatial period of the external forcing is the same as that of the traveling waves, i.e., $q_{f}=q_{c}$. Hereafter, the parameters are fixed as $\tau=1.6$ and $\gamma_{2}=0.16$. Because we set the value of $\tau$ close to the critical value $\tau_{c}=1.46$, throughout this paper, we assume that the actual wave number of the traveling wave does not differ much from $q_{c}$. The numerical simulations discussed in this and the next section were carried out as follows. The system in one dimension was divided into $N=128$ cells, with the cell size $\delta x=20 \pi / N$. The linear dimension of the system was, therefore, $N \times \delta x=20 \pi \approx 63$, which is commensurate with the spatial period $(\approx 7)$ of the traveling wave. Periodic boundary conditions were imposed at the system boundaries. The forward Euler method was used to solve the partial differential equations. In most simulations, the time increment $\delta t$ was chosen as $\delta t=0.001$.

The dynamics of the traveling waves obtained by altering the external frequency and the force strength $\epsilon$ are summarized in Fig. 3 [27]. These results were obtained by extracting the asymptotic behavior of the traveling wave after imposing the external force. In the region indicated by the symbols + , the traveling wave is entrained to the external force, so that it propagates at the velocity $\Omega / q_{c}$. When the frequency $\Omega$ of the external forcing is far from the intrinsic frequency $\omega_{c}$, as in the region indicated by the diamonds, entrainment breaks down; that is, the traveling wave and the 


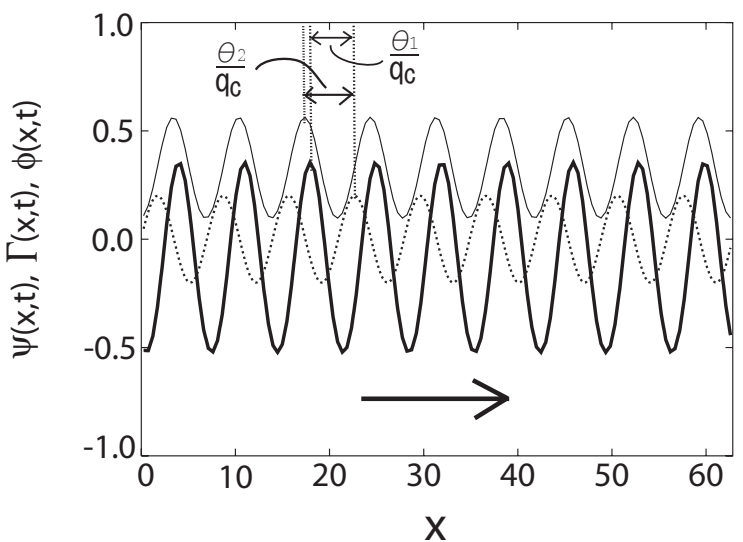

FIG. 4. Spatial profiles of $\psi(x, t)$ (thick solid curve), $\phi(x, t)$ (thin solid curve), and $\Gamma(x, t)$ for $\gamma_{2}=0.16, \tau=1.6, \Omega=0.07$, and $\epsilon$ $=0.002$. The phase differences $\theta_{1}$ and $\theta_{2}$ are also indicated. The scale of $\Gamma$ is multiplied by 100 .

external forcing propagate at different velocities. For example, when the frequency $\Omega$ is substantially larger than $\omega_{c}$, the traveling wave with velocity $\omega_{c} / q_{c}$, cannot keep up with the propagating external forcing with velocity $\Omega / q_{c}$ and hence the external wave passes through the traveling wave. Because the spatial period of the external forcing is the same as that of the traveling wave, this occurs simultaneously for all the wave trains, i.e., uniformly in space. During the interval that the external wave passes through the traveling wave, the latter is modulated such that the amplitude and the propagation velocity are decreased. It is readily understood that this modulation occurs periodically with a period approximately given by $2 \pi /\left|\Omega-\omega_{c}\right|$.

The region of complete entrainment has been investigated using the amplitude equation of a propagating wave [27]. The theoretical boundaries indicated by the solid lines in Fig. 3 are consistent with the simulations except for $\epsilon=0.009$ where the expansion in powers of $\epsilon$ employed in the theory becomes worse. The detailed behavior in the vicinity of the stability boundary, given by $\Omega \approx 0.04$ and $\epsilon=0.004$, which was not examined in Ref. [27], is discussed at the end of this section.

(a)

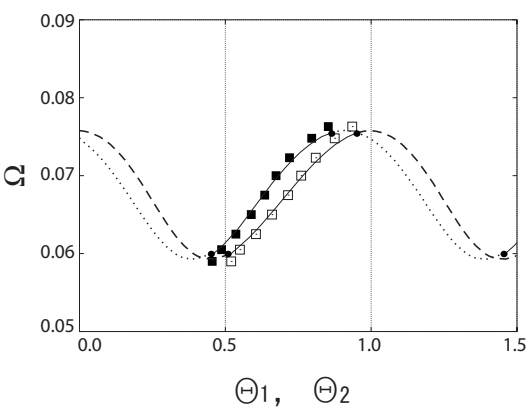

(b)

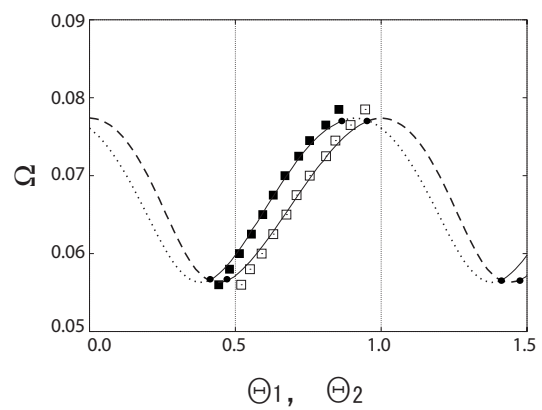

Here we focus our attention on the wave dynamics in the case of complete entrainment. That is, we are concerned with the phase of the propagating waves. Figure 4 displays the spatial profile of the variables $\psi(x, t)$ and $\phi(x, t)$ and the external force $\Gamma(x, t)$ at an arbitrary time. The phase difference between $\psi$ and $\Gamma$ depends on the external frequency $\Omega$ for a given value of the strength $\epsilon$. Here, the phases are defined through

$$
\begin{aligned}
& \psi(x, t)=\hat{\psi}\left[q_{c} x-\Omega t+\theta_{1}(t)\right], \\
& \phi(x, t)=\hat{\phi}\left[q_{c} x-\Omega t+\theta_{2}(t)\right],
\end{aligned}
$$

where $\hat{\psi}(x)$ and $\hat{\phi}(x)$ are periodic functions of period $2 \pi / q_{c}$.

Note that the condition of complete entrainment is characterized by a well-defined relationship between the external frequency $\Omega$ and the phase difference between the external force and the entrained oscillations [2,30]. When $\Omega$ increases, the phase difference is expected to increase, because the wave is forced to follow the external forcing, even though the intrinsic velocity $\omega_{c}$ of the wave is smaller. Hence the wave is slowed by the external force. When $\Omega$ decreases, the phase difference becomes smaller. Therefore we expect the relation

$$
\frac{d \theta_{1}}{d \Omega}>0,
$$

where we have regarded $\theta_{1}$ as a function of $\Omega$. The relations between the asymptotic phase differences $\Theta_{1}=\theta_{1} /(2 \pi)$ or $\Theta_{2}=\theta_{2} /(2 \pi)$ and the external frequency $\Omega$ is shown in Figs. 5(a) -5 (c) for $\epsilon=0.002, \epsilon=0.0025$, and $\epsilon=0.004$, respectively. It is evident that the phase differences $\theta_{1}$ (black squares) and $\theta_{2}$ (white squares) increase as the external frequency $\Omega$ is increased. Furthermore, the simulation results are quite consistent with the theoretical results, indicated by the lines.

The frequency $\Omega$ is almost sinusoidal as a function of $\Theta_{1}$ and $\Theta_{2}$, and the amplitude that increases with the external strength $\epsilon$. In fact, we find the approximate relation 0.059 $<\Omega<0.076$ for $\epsilon=0.002,0.056<\Omega<0.079$ for $\epsilon=0.0025$, and $0.041<\Omega<0.082$ for $\epsilon=0.004$. These are consistent with the simulation results displayed in Fig. 3. Note that the

FIG. 5. Relation between the phase and $\Omega$ for (a) $\epsilon=0.002$, (b) $\epsilon=0.0025$, and (c) $\epsilon=0.004$. The black squares represent the $\Theta_{1}$ $=\theta_{1} /(2 \pi)$ dependence, whereas the white squares represent the $\Theta_{2}=\theta_{2} /(2 \pi)$ dependence. The solid curves are the stable solutions obtained from Eqs. (38) and (39). The dotted (broken) curve is the unstable solution of $\theta_{1}\left(\theta_{2}\right)$. The small dots indicate the boundary between the stable and unstable solutions. Note that the unstable solution does not exist below $\Omega=0.04$ for (c) $\epsilon=0.004$, as indicated by the arrows. 
$\Omega-\Theta$ relation exhibits anomalous behavior near $\Omega=0.04$ for $\epsilon=0.004$, as shown in Fig. 5(c). That is, the theoretically determined curves of $\Theta_{1}$ and $\Theta_{2}$ regarded as functions of $\Omega$ are discontinuous at a point near $\Omega=0.04$ as indicated by the arrows in Fig. 5(c). This means that the entrained wave does not exist for $\epsilon=0.004$ when $\Omega<0.04$. This result is consistent with the results in Fig. 3, where one can see clearly that the point at $(\epsilon, \Omega)=(0.004,0.04)$ is outside the entrained region. We present an analysis of this singular behavior in Sec. V.

\section{FEEDBACK CONTROL}

The feedback effect of the propagating waves is investigated using the equations

$$
\begin{aligned}
\frac{\partial \psi}{\partial t}= & \nabla^{2}\left(-\nabla^{2} \psi-\tau \psi+\psi^{3}\right)+a_{1} \psi+a_{2} \phi+a_{3} \\
& +F[\psi(x-\delta, t)-\bar{\psi}], \\
\frac{\partial \phi}{\partial t}= & b_{1} \psi+b_{2} \phi+b_{3}+F[\psi(x-\delta, t)-\bar{\psi}],
\end{aligned}
$$

where the coefficient $F$ is the strength of the feedback term and $\bar{\psi}$ is the spatial average of $\psi$. Note that we have added a term given by the profile of $\psi$ but with a space shift $\delta$. We examine how the propagating waves are affected by the feedback term by altering the strength $F$ and the spatial shift $\delta$.

As mentioned in Sec. I, our main purpose is to investigate the dynamics of traveling waves under feedback-mediated spatiotemporal control in distributed systems. In the previous studies of such feedback control, time-delayed feedback was employed to derive a well-defined relationship between the external frequency $\Omega$ and the phase difference between the external force and the entrained oscillations [2,30]. In Eqs. (24) and (25), by contrast, we have employed a spatial shift to exercise control. Note that, in contrast to the time-delay control frequently used, such a spatial shift can be either positive or negative. Another reason that we employ this type of control is that it is easily treated both numerically and analytically. Contrastingly, simulations of systems with timedelayed control that investigate asymptotic steady state behavior are time consuming even in one dimension. The phase dynamic approach presented in the following sections is simpler in the case of spatial-shift control than in the case of time-delayed control. We show below that some unexpected interesting dynamics appear even in the case of spatial-shift control.

We solved Eqs. (24) and (25) numerically in one dimension. Initially, we started with the set of equations with $F$ $=0$. After a wave propagating to the right-hand side developed, we turned on the feedback term, setting $F$ to some finite value. The asymptotic traveling velocity $V$ is evaluated as a function of the spatial shift $\delta$ for a given value of $F$. The relation between the frequency $\Omega_{F B}=q_{c} V$ of the controlled wave and the normalized phase shift $\Delta=q_{c} \delta / 2 \pi$ is displayed in Figs. 6(a)-6(d) for several values of $F$.

It is evident from Figs. 6(a)-6(d) that the frequency depends sinusoidally on the shift for small values of the feed- back strength $F$, with an amplitude that increases with $F$. In contrast to the case of external forcing, in this case, a stable wave exists both for $d \Omega_{F B} / d \Delta<0$ and $d \Omega_{F B} / d \Delta>0$. For $F<0.0057$, the frequency is positive, which means that the wave propagates to the right-hand side under the feedback control. What is most noteworthy here is that the frequency jumps discontinuously at some value of $\Delta$ and changes sign when $F$ is large, as shown in Figs. 6(c) and 6(d). This reflects the reversal of the propagation direction of the wave train. An example of such reversal is displayed in Fig. 7 for $F$ $=0.0090$ and $\Delta=0.35$. Before the feedback control is turned on, at $t=600$, the wave propagates to the right-hand side. However, after $t=600$, the direction of propagating switches to the left-hand side.

Figures $8(\mathrm{a})-8(\mathrm{~d})$ display the propagating frequency $\Omega_{F B}$ as a function of the phase shift $\Delta$ for several values of $F$. In Fig. 8(a) it is seen that when the value of $\Delta$ is small, the frequency $\Omega_{F B}$ increases monotonically with the strength $F$. In fact, if the value $\Delta$ satisfies $d^{2} \Omega_{F B} / d \Delta^{2}<0$, then $\Omega_{F B}$ is an increasing function of $F$, since the amplitude of the sinusoidal variation of $\Omega_{F B}$ increases as a function of $F$, as can be seen in Figs. 6(a) and 6(b). When $\Delta$ exceeds a certain value, a discontinuity appears, as shown in Figs. 8(b) and $8(\mathrm{c})$. Then, for $\Delta>0.5$, the frequency $\Omega_{F B}$ again becomes positive, as shown in Figs. 6(c) and 6(d), and thus no discontinuity appears in Fig. 8(d) for $\Delta=0.63$. The parameter region in which the propagation direction reverses is displayed in Fig. 9 in the $F-\Delta$ plane. This region is periodic in $\Delta$, as expected.

\section{PHASE DYNAMICS FOR EXTERNAL FORCING}

In this section we show that both external forcing and the feedback control studied in the preceding section can be understood in terms of the phase dynamics approach. Below, we derive the amplitude equations and the phase equations in the case of external forcing. Near the Hopf bifurcation, where the nonlinear effects are small, we can write the variables as

$$
\begin{aligned}
& \psi=\psi_{0}+\psi_{1}(t) \cos \left[q_{c} x-\Omega t+\theta_{1}(t)\right], \\
& \phi=\phi_{0}+\phi_{1}(t) \cos \left[q_{c} x-\Omega t+\theta_{2}(t)\right],
\end{aligned}
$$

where the time-evolution equations for the amplitudes $\psi_{1}(t)$ and $\phi_{1}(t)$ and the phases $\theta_{1}(t)$ and $\theta_{2}(t)$ are to be derived. Substituting Eqs. (26) and (27) into Eqs. (17) and (18), multiplying Eq. (17) by $\sin \left[q_{c} x-\Omega t+\theta_{1}(t)\right]$ and Eq. (18) by $\sin \left[q_{c} x-\Omega t+\theta_{2}(t)\right]$, and integrating over one spatial period, we obtain

$$
\begin{aligned}
& \frac{d \theta_{1}}{d t}=\Omega-\frac{a_{2} \phi_{1}}{\psi_{1}} \sin \left(\theta_{1}-\theta_{2}\right)-\frac{\epsilon}{\psi_{1}} \sin \theta_{1}, \\
& \frac{d \theta_{2}}{d t}=\Omega+\frac{b_{1} \psi_{1}}{\phi_{1}} \sin \left(\theta_{1}-\theta_{2}\right)-\frac{\epsilon}{\phi_{1}} \sin \theta_{2} .
\end{aligned}
$$

Similarly, multiplying Eqs. (17) and (18) by $\cos \left[q_{c} x-\Omega t\right.$ $\left.+\theta_{1}(t)\right]$ and $\cos \left[q_{c} x-\Omega t+\theta_{2}(t)\right]$, respectively, and integrating 
(a)

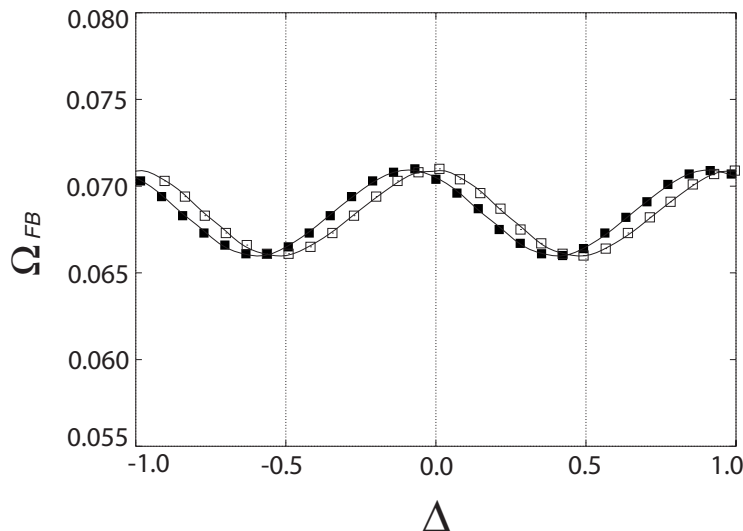

(c)

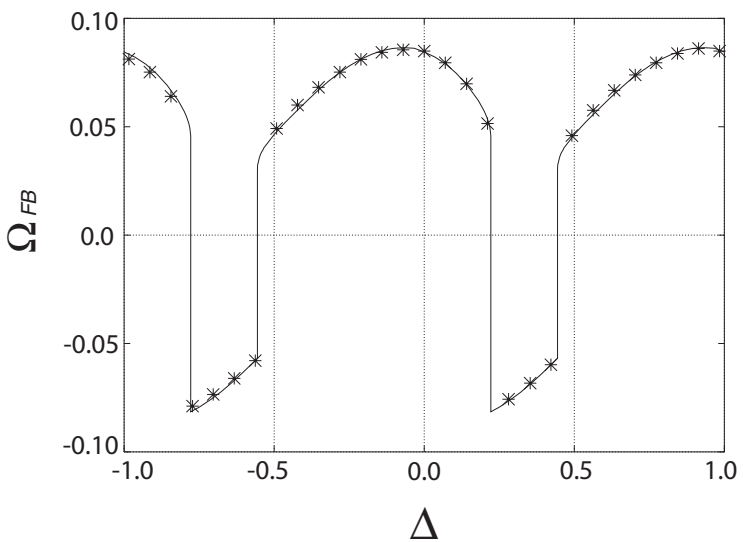

(b)

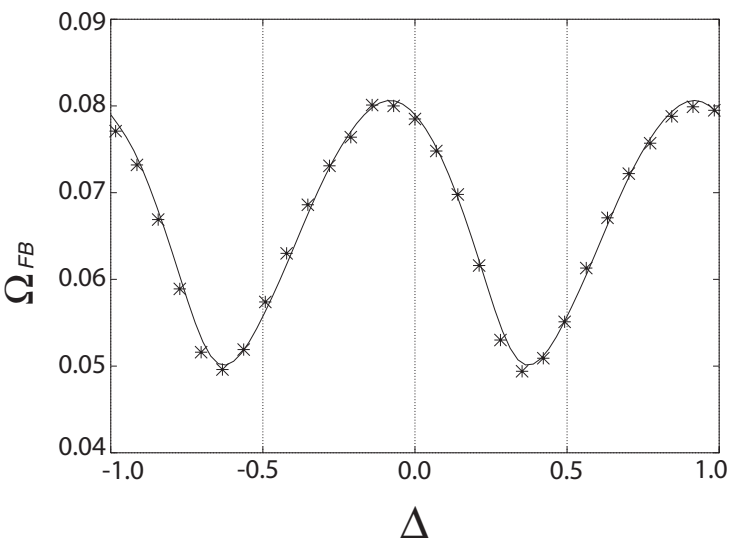

(d)

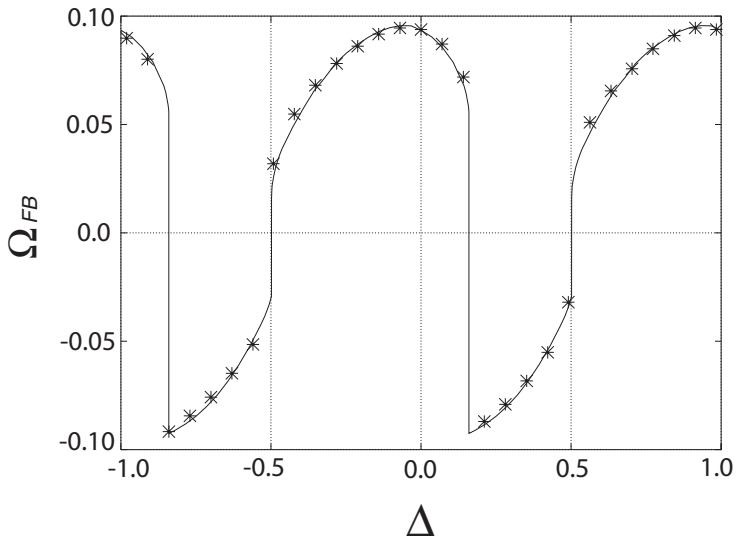

FIG. 6. Relation between the frequency $\Omega_{F B}$ and the phase shift $\Delta=q_{c} \delta / 2 \pi$ for (a) $F=0.0010$, (b) $F=0.0057$, (c) $F=0.0090$, and (d) $F=0.0150$. In (a), the simulation results are represented by the black squares for $\Delta$, together with the phase $\theta_{2} / 2 \pi$ (white squares) for comparison. In (c) and (d) the results of simulations for $\Delta$ are represented by the symbols *, which should be compared with the theoretical results given in Eqs. (50) and (51) (solid curves).

over one spatial period, we obtain the set of equations for the amplitudes $\psi_{1}$ and $\phi_{1}$,

$$
\begin{gathered}
\frac{d \psi_{1}}{d t}=\left[q_{c}^{4}+q_{c}^{2}\left(\tau-\tau_{c}\right)\right] \psi_{1}+a_{1} \psi_{1}-\frac{3}{4} q_{c}^{2} \psi_{1}^{3} \\
+a_{2} \phi_{1} \cos \left(\theta_{1}-\theta_{2}\right)+\epsilon \cos \theta_{1} \\
\frac{d \phi_{1}}{d t}=b_{1} \psi_{1} \cos \left(\theta_{1}-\theta_{2}\right)+b_{2} \phi_{1}+\epsilon \cos \theta_{2}
\end{gathered}
$$

It should be noted that the set of Eqs. (28)-(31) has an invariance under $\theta_{n} \rightarrow \theta_{n}+c \quad(n=1$ and 2) with an arbitrary constant $c$ when the external field is absent. This neutral stability of the phases is a general property originating from the translational invariance of time (see, e.g., [2]). An immediate consequence is that there is a mode of the phase variables, whose eigenvalue is equal to zero in the linear stability analysis of the time-independent solutions of the above set of equations. On the other hand, the amplitudes $\psi_{1}$ and $\phi_{1}$ have finite relaxation rates. When the time-dependent small external field is present as in Eqs. (17) and (18), the translational

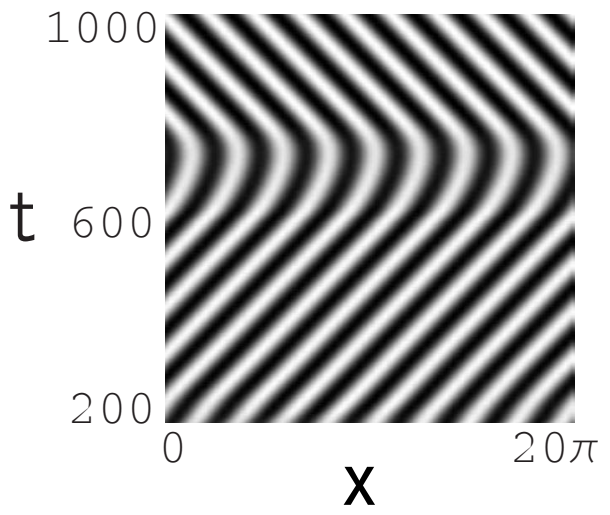

FIG. 7. Space-time plot of the propagating wave for $F=0.009$ and $\Delta=0.35$. The gray scale indicates the magnitude of the variable $\psi$. The feedback forcing is changed from 0 to a finite value at $t$ $=600$. Before that time, the wave propagates to the right-hand side. The feedback forcing eventually causes the direction of propagation to switch to the left-hand side. 
(a)

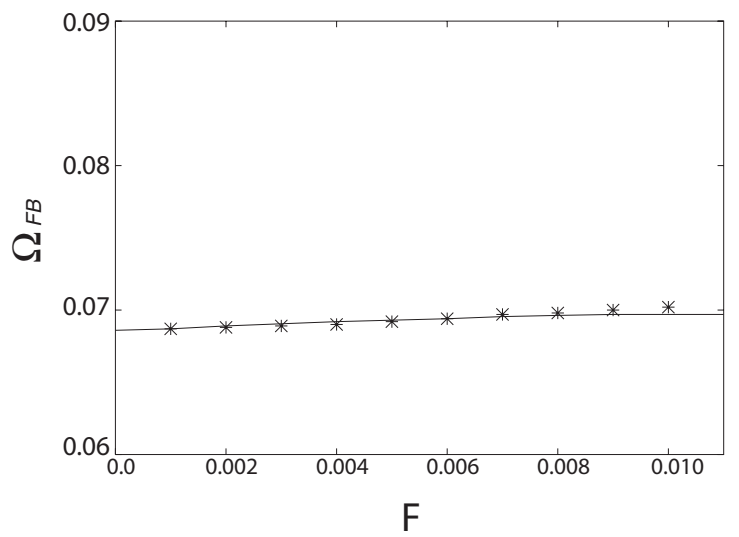

(c)

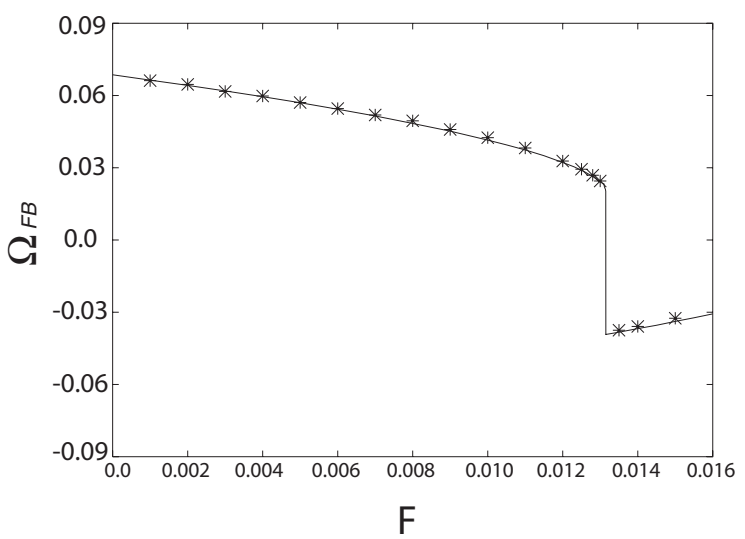

(b)

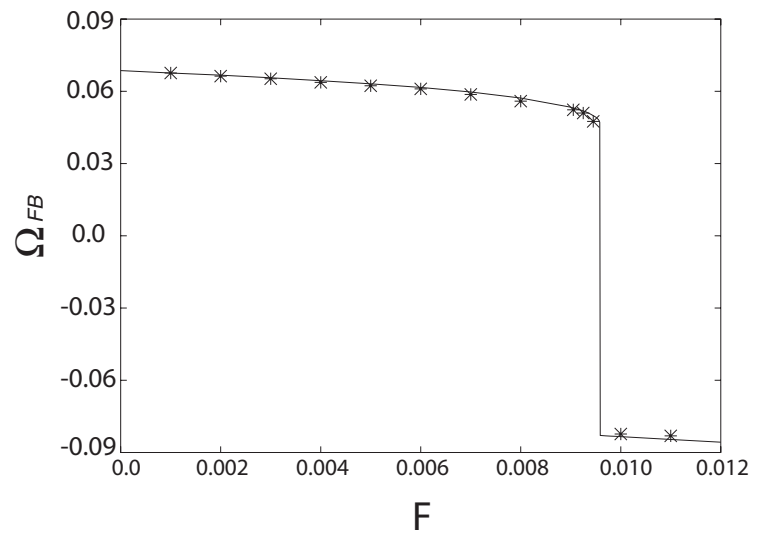

(d)

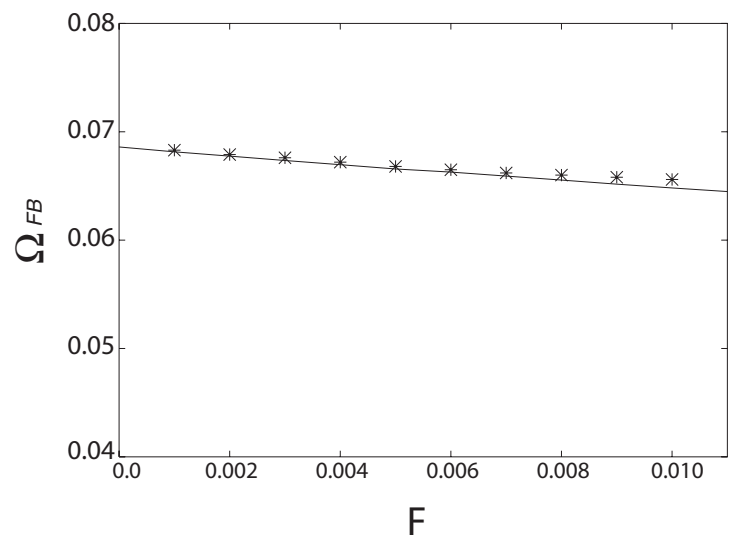

FIG. 8. $F$ dependence of $\Omega_{F B}$ for $\Delta \approx 0.14$, (b) $\Delta \approx 0.21$, (c) $\Delta \approx 0.49$, and (d) $\Delta \approx 0.63$. The results of the simulations, represented by the symbols *, are compared with the theoretical results given by Eqs. (50) and (51) (solid curves).

invariance is violated and the zero eigenvalue turns out to be finite of the order of $\epsilon$ whereas the characteristic time of the amplitudes is of the order of unity. This difference of the two time scales enables us to employ the adiabatic elimination of the amplitudes $\psi_{1}$ and $\phi_{1}$ by setting $d \psi_{1} / d t=d \phi_{1} / d t=0$ in Eqs. (30) and (31). The same procedure can be applied to the case of the feedback forcing in Sec. VI.

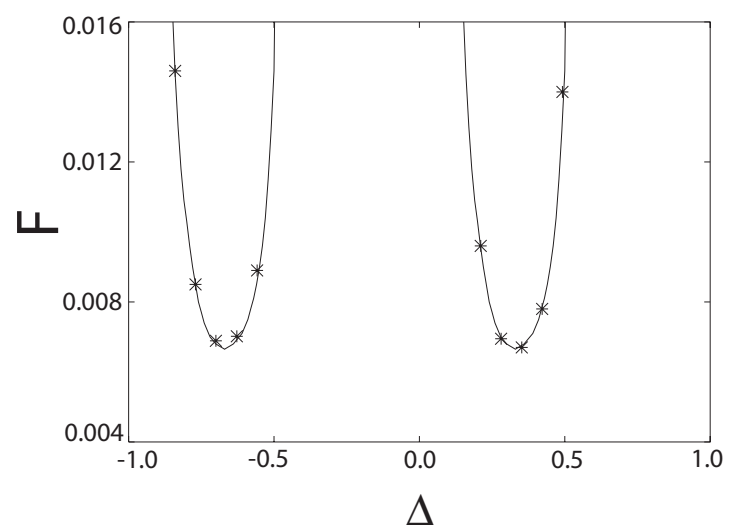

FIG. 9. Phase diagram for the reversal of the propagation direction. The results of the simulations are represented by the symbols *, whereas the theoretically derived boundary, obtained from Eqs. (50) and (51), is represented by the solid line.
Actually, the above-mentioned elimination can be carried out systematically by a perturbation expansion in terms of the strength of the external forcing $\epsilon$. This is done by first writing

$$
\begin{aligned}
& \psi_{1}=\psi_{1}^{(0)}+\psi_{1}^{(1)}, \\
& \phi_{1}=\phi_{1}^{(0)}+\phi_{1}^{(1)} .
\end{aligned}
$$

Then, we readily obtain the following expression of the zeroth-order solution:

$$
\begin{gathered}
\frac{3}{4}\left(\psi_{1}^{(0)}\right)^{2}=q_{c}^{2}+\tau-\tau_{c}+\frac{1}{q_{c}^{2}}\left(a_{1}-\frac{a_{2} b_{1}}{b_{2}}\left[\cos \left(\theta_{1}-\theta_{2}\right)\right]^{2}\right) \\
\phi_{1}^{(0)}=\left(-\frac{b_{1}}{b_{2}} \cos \left(\theta_{1}-\theta_{2}\right)\right) \psi_{1}^{(0)} .
\end{gathered}
$$

Here, the positive solution for $\psi_{1}^{(0)}$ is chosen for consistency with the simulations. The first-order solution is given by

$$
\psi_{1}^{(1)}=\frac{2 \epsilon}{3 q_{c}^{2}\left(\psi_{1}^{(0)}\right)^{2}}\left(\cos \left(\theta_{1}\right)-\frac{a_{2}}{b_{2}} \cos \left(\theta_{2}\right) \cos \left(\theta_{1}-\theta_{2}\right)\right),
$$


(a)

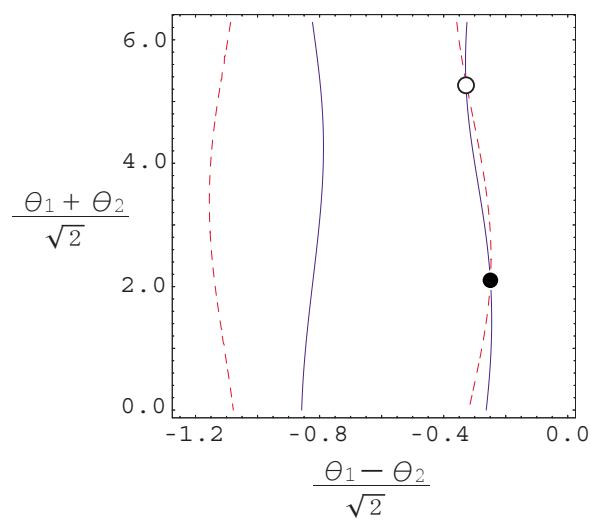

$$
\phi_{1}^{(1)}=\frac{\phi_{1}^{(0)}}{\psi_{1}^{(0)}} \psi_{1}^{(1)}-\frac{\epsilon}{b_{2}} \cos \left(\theta_{2}\right) .
$$

Substituting Eqs. (34)-(37) into (28) and (29), we obtain a closed set of equations for the phases,

$\frac{d \theta_{1}}{d t}=\Omega-\frac{\epsilon}{\psi_{1}^{(0)}} \sin \left(\theta_{1}\right)-a_{2} \frac{\phi_{1}^{(0)}}{\psi_{1}^{(0)}}\left(1+\frac{\phi_{1}^{(1)}}{\phi_{1}^{(0)}}-\frac{\psi_{1}^{(1)}}{\psi_{1}^{(0)}}\right) \sin \left(\theta_{1}-\theta_{2}\right)$,

$\frac{d \theta_{2}}{d t}=\Omega-\frac{\epsilon}{\phi_{1}^{(0)}} \sin \left(\theta_{2}\right)+b_{1} \frac{\psi_{1}^{(0)}}{\phi_{1}^{(0)}}\left(1+\frac{\psi_{1}^{(1)}}{\psi_{1}^{(0)}}-\frac{\phi_{1}^{(1)}}{\phi_{1}^{(0)}}\right) \sin \left(\theta_{1}-\theta_{2}\right)$.

There is a set of equilibrium solutions to Eqs. (38) and (39) for a finite range of $\Omega$. The theoretical results are plotted in Fig. 5 in comparison with the results of the simulations. Note that the stable region of the solutions is consistent with the simulations. Moreover, even the existence of the discontinuity of the solution near $\Omega=0.04$ for $\epsilon=0.004$, as seen in Fig. 5 (c), can be accounted for quantitatively by this analysis. In order to elucidate this, the null clines of Eqs. (38) and (39) for $\epsilon=0.004$ are plotted in Fig. 10, where the linear combinations of the variables $\theta_{1}$ and $\theta_{2}$ are used for convenience. In the case of large $\Omega(\Omega=0.06)$, depicted in Fig. 10(a), there is a pair of equilibrium solutions, one stable and one unstable. However, this pair of solutions disappears at some value near $\Omega=0.041$, as shown in Fig. 10(b). This is the reason that the theoretically determined lines do not exist below $\Omega=0.04$ in Fig. 5(c).

\section{PHASE DYNAMICS FOR FEEDBACK CONTROL}

Now we formulate the phase dynamics for the case of feedback control. The method here is similar to that in the case of external forcing outlined in the preceding section. In this case, we write

$$
\begin{aligned}
& \psi=\psi_{0}+\psi_{1}(t) \cos \left[q_{c} x-\omega_{c} t+\theta_{1}(t)\right] \\
& \phi=\phi_{0}+\phi_{1}(t) \cos \left[q_{c} x-\omega_{c} t+\theta_{2}(t)\right] .
\end{aligned}
$$

Substituting these into Eqs. (24) and (25), we obtain (b)

$$
\omega_{c}-\frac{d \theta_{1}}{d t}=\frac{a_{2} \phi_{1}}{\psi_{1}} \sin \left(\theta_{1}-\theta_{2}\right)+F \sin \left(q_{c} \delta\right)
$$

$$
\begin{aligned}
& \omega_{c}-\frac{d \theta_{2}}{d t}=-\frac{b_{1} \psi_{1}}{\phi_{1}} \sin \left(\theta_{1}-\theta_{2}\right)+\frac{F \psi_{1}}{\phi_{1}} \sin \left[q_{c} \delta-\left(\theta_{1}-\theta_{2}\right)\right] \\
& \frac{d \psi_{1}}{d t}=\left[q_{c}^{4}+q_{c}^{2}\left(\tau-\tau_{c}\right)\right] \psi_{1}+a_{1} \psi_{1}-\frac{3}{4} q_{c}^{2} \psi_{1}^{3} \\
& +a_{2} \phi_{1} \cos \left(\theta_{1}-\theta_{2}\right)+F \psi_{1} \cos \left(q_{c} \delta\right), \\
& \frac{d \phi_{1}}{d t}=b_{1} \psi_{1} \cos \left(\theta_{1}-\theta_{2}\right)+b_{2} \phi_{1}+F \psi_{1} \cos \left[q_{c} \delta-\left(\theta_{1}-\theta_{2}\right)\right]
\end{aligned}
$$

As in the case treated above, the amplitudes $\psi_{1}$ and $\phi_{1}$ which evolve slowly in time are eliminated adiabatically by setting $d \psi_{1} / d t=d \phi_{1} / d t=0$ and obtained through a perturbative expansion with respect to $\epsilon$. It is obvious that the zeroth-order solution, $\psi_{1}^{(0)}$ and $\phi_{1}^{(0)}$, should be the same as that given in Eqs. (34) and (35),

$$
\begin{gathered}
\frac{3}{4}\left(\psi_{1}^{(0)}\right)^{2}=q_{c}^{2}+\tau-\tau_{c}+\frac{1}{q_{c}^{2}}\left(a_{1}-\frac{a_{2} b_{1}}{b_{2}}\left[\cos \left(\theta_{1}-\theta_{2}\right)\right]^{2}\right), \\
\phi_{1}^{(0)}=-\frac{b_{1} \psi_{1}^{(0)}}{b_{2}} \cos \left(\theta_{1}-\theta_{2}\right) .
\end{gathered}
$$

The first-order corrections, $\psi_{1}^{(1)}$ and $\phi_{1}^{(1)}$, are given by

$$
\begin{aligned}
\psi_{1}^{(1)}= & \frac{2 F}{3 q_{c}^{2} \psi_{1}^{(0)}}\left(\cos \left(q_{c} \delta\right)-\frac{a_{2}}{b_{2}} \cos \left[q_{c} \delta-\left(\theta_{1}-\theta_{2}\right)\right]\right. \\
& \left.\times \cos \left(\theta_{1}-\theta_{2}\right)\right) \\
\phi_{1}^{(1)}= & \frac{\phi_{1}^{(0)}}{\psi_{1}^{(0)}} \psi_{1}^{(1)}-\frac{F \psi_{1}^{(0)}}{b_{2}} \cos \left[q_{c} \delta-\left(\theta_{1}-\theta_{2}\right)\right] .
\end{aligned}
$$

In this way, from Eqs. (42), (43), and (46)-(49), we obtain 


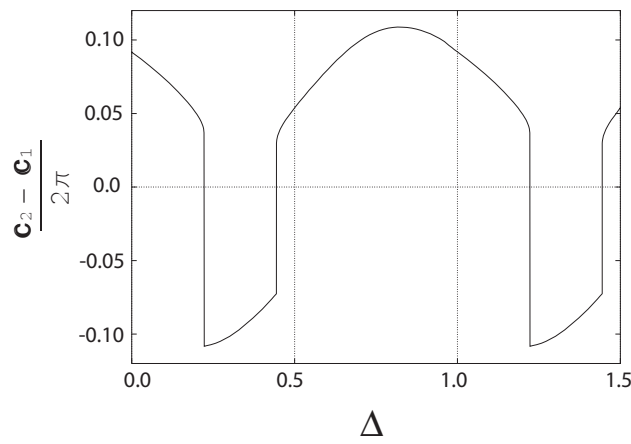

FIG. 11. $c_{2}-c_{1}$ as a function of $\Delta$ for $F=0.009$.

$\omega_{c}-\frac{d \theta_{1}}{d t}=F \sin \left(q_{c} \delta\right)+a_{2} \frac{\phi_{1}^{(0)}}{\psi_{1}^{(0)}}\left(1+\frac{\phi_{1}^{(1)}}{\phi_{1}^{(0)}}-\frac{\psi_{1}^{(1)}}{\psi_{1}^{(0)}}\right) \sin \left(\theta_{1}-\theta_{2}\right)$,

$$
\begin{aligned}
\omega_{c}-\frac{d \theta_{2}}{d t}= & \frac{F \psi_{1}^{(0)}}{\phi_{1}^{(0)}} \sin \left[q_{c} \delta-\left(\theta_{1}-\theta_{2}\right)\right] \\
& -b_{1} \frac{\psi_{1}^{(0)}}{\phi_{1}^{(0)}}\left(1+\frac{\psi_{1}^{(1)}}{\psi_{1}^{(0)}}-\frac{\phi_{1}^{(1)}}{\phi_{1}^{(0)}}\right) \sin \left(\theta_{1}-\theta_{2}\right) .
\end{aligned}
$$

We can show that the solutions of Eqs. (50) and (51) in the limit $t \rightarrow \infty$ take the form $\theta_{1}=\omega t+c_{1}$ and $\theta_{2}=\omega t+c_{2}$, where the constants $\omega$ and $c_{2}-c_{1}$ are evaluated numerically. An example of this solution is plotted in Fig. 11. The frequency of the propagating wave under feedback control is given by

$$
\Omega_{F B}=\omega_{c}-\omega .
$$

The relation between $\Omega_{F B}$ and the phase shift variable defined by $\Delta=\left(q_{c} \delta\right) /(2 \pi)$ is depicted by the solid line in Figs. 6(a)-6(d). The $F$ dependence is shown in Figs. 8(a)-8(d). It is found that these theoretical results agree almost completely with the simulations.

Next, we clarify the reason that $\Omega_{F B}$ becomes negative discontinuously as a function of $\Delta$ for large values of $F$. First, note that the right-hand sides of Eqs. (50) and (51) are functions of $\theta_{2}-\theta_{1}$. Therefore, combining these two equations we obtain a closed equation for $\theta_{2}-\theta_{1}$. Figures 12(a)-12(c) plot $d\left(\theta_{2}-\theta_{1}\right) / d t$ as functions of $\theta_{2}-\theta_{1}$ for $\Delta$ $=0.07, \Delta=0.35$, and $\Delta=0.63$. For $\Delta=0.07$, there are two stable and one unstable solutions, as shown in Fig. 12(a). Since we have chosen a wave propagating to the right-hand side, $\theta_{2}-\theta_{1}$ is always positive. Therefore, the positive stable solution is approached asymptotically. However, when $\Delta$ is increased, the system becomes monostable, leaving only a stable negative solution, as shown in Fig. 12(b). Hence, the value of $\omega_{c}-\omega$ exhibits a discontinuous jump when the transition from a bistable to a monostable system occurs. If the value of $\Delta$ is increased further, the system again becomes bistable, and then eventually monostable once more, but this time with a positive solution for $\theta_{2}-\theta_{1}$.

The behavior described above can be understood completely by the following argument. First, let us write the equation for $\theta_{2}-\theta_{1}$ as

$$
\frac{d\left(\theta_{2}-\theta_{1}\right)}{d t}=a_{0} F \sin (2 \pi \Delta)+g\left(\theta_{2}-\theta_{1}\right)
$$

where $a_{0}$ and $g(x)$ contain $F$ and $\Delta$, as can be seen from Eqs. (50) and (51). It is important to note that $g(x)$ is an odd function when the feedback control is absent (i.e., when $F$ $=0$ ). This is because the nonlinear dissipative wave considered here possesses a parity symmetry; that is, for every wave propagating in one direction, there is a corresponding wave propagating in the other direction obtained from it through spatial reflection. Then, clearly, the phase difference $\theta$ given by Eq. (11) for the right-propagating as opposite sign for the left-propagating wave. This means that $g(x)$ should take the following form:

$$
g\left(\theta_{2}-\theta_{1}\right)=a_{1}\left(\theta_{2}-\theta_{1}\right)-a_{3}\left(\theta_{2}-\theta_{1}\right)^{3}
$$

for small values of $\theta_{2}-\theta_{1}$ with positive coefficients $a_{1}$ and $a_{3}$. Now in the case that $F \neq 0$, these coefficients depend on $\Delta$. However as long as $F$ is small, we can ignore such dependence. Then, Eq. (53) with (54) reveals that the system is bistable when $\Delta$ or $F$ is small, and then becomes, in succession, monostable with a negative solution for $a_{0}<0$, bistable and again monostable with a positive solution, as $\Delta$ is increased. This result accounts for all of the results of the numerical simulations.

\section{DISCUSSION}

In this paper, we have investigated the dynamics of nonlinear dissipative waves under external and feedback controls through both numerical simulations and the phase dynamics (a)

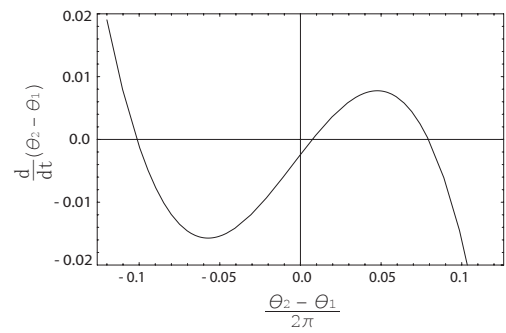

(b)

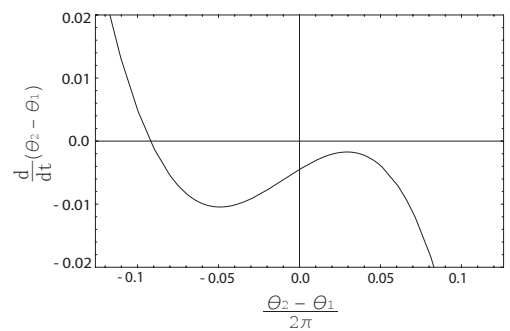

(c)

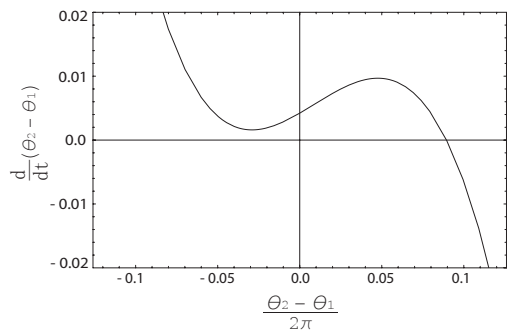

FIG. 12. $d\left(\theta_{2}-\theta_{1}\right) / d t$ as a function of $\theta_{2}-\theta_{1}$ for $F=0.009$ and for (a) $\Delta=0.07$, (b) $\Delta=0.35$, and (c) $\Delta=0.63$. 
approach. In order to emphasize the aspects of the present results, we now briefly review the theory for the entrainment of a limit cycle oscillation under external periodic modulations. When the limit cycle can be represented in terms of the phase variable as $d \phi / d t=\omega$, and the frequency $\omega$ of the limit cycle is a constant close to that of the external frequency $\Omega$, the phase difference $\theta=\Omega t-\phi$ between the oscillator and the external force obeys the equation (see, e.g., [30])

$$
\frac{d \theta}{d t}=\Omega-\omega+f(\theta),
$$

where $f$ is a $2 \pi$-periodic function of $\theta$. This equation implies that entrainment occurs when $|\Omega-\omega| \leq|f(\phi)|$. [The phase $\theta$ is defined consistently with those in Eqs. (21) and (22).] It is readily verified that the stable solution of Eq. (55) satisfies $d \Omega / d \theta>0$.

It is now seen that there is an essential difference between the theory of entrainment in nonlinear propagating waves and the formulation of limit cycle oscillation sketched above. As shown in Eqs. (38) and (39) for external forcing and Eqs. (50) and (51) for feedback control in the types of the systems studied here, two phase variables must be introduced to properly describe the dynamics. If we had fixed the difference between the two phases as a constant and derived the equation for only one variable, we could not have obtained results from this phase dynamics treatment consistent with the simulation results. This point can be understood from two theoretical observations. One is the existence and nonexistence of a pair of solutions in the coupled phase equations, as shown in Figs. 10(a) and 10(b). Note that the horizontal axes of these figures represent the phase difference $\theta_{1}-\theta_{2}$. Thus, if the phase difference were set to a constant $a$ priori, the entrainment of the wave to the external forcing would not be obtained properly. The other is the reversal of the propagation direction, depicted in Figs. 12(a)-12(c), in which the time evolution of the phase difference $\theta_{1}-\theta_{2}$ plays an essential role.

We have thus found that, as in the case of lumped oscillator systems, feedback control is capable of stabilizing certain types of behavior in some dynamical regimes that are unstable in the case that periodic forcing is applied. In addition, the feedback control investigated here, which employs a spatial shift of the profile of a measured variable, creates very unusual dynamical behavior of the model system resulting in the reversal of the direction of the wave propagation.

The present investigation is focused on the case in which the spatial period of the external or feedback signal is the same as that of the propagating wave. We have shown that the phase dynamic approach is quite successful in treating such systems, and indeed, almost all the phenomena found in the numerical simulations can be accounted for within the phase dynamics framework. In the case that the periods of the external forcing and the propagating wave are incommensurate, however, it is believed that the dynamics would be more complicated and that the system would exhibit a much greater variety of patterns. Some preliminary findings for systems of this type are presented in [28]. An extension of the theory used there will be reported in the future.

\section{ACKNOWLEDGMENTS}

One of the authors (T.O.) would like to thank Professor Helmut Brand for valuable discussions and encouragement. This work was supported by a Grant-in-Aid for the 21 st Century COE "Center for Diversity and Universality in Physics" and a Grant-in-Aid for the superior area "Soft Matter Physics", both from the Ministry of Education, Culture, Sports, Science and Technology (MEXT) of Japan, and by Grant No. SFB 555 from the Deutsche Forschungsgemeinschaft.
[1] G. Nicolis and I. Prigogine, Self-Organization in Non Equilibrium Systems (Wiley, New York, 1977).

[2] Y. Kuramoto, Chemical Oscillations, Waves and Turbulence (Springer, Berlin, Heidelberg, 1984).

[3] Wave and Patterns in Biological and Chemical Excitable Media, edited by V. Krinsky and H. Swinney (North-Holland, Amsterdam, 1991).

[4] M. C. Cross and P. C. Hohenberg, Rev. Mod. Phys. 65, 851 (1993).

[5] Chemical Waves and Patterns, edited by R. Kapral and K. Showalter (Kluwer, Dordrecht, 1995).

[6] E. Schöll, Nonlinear Spatio-Temporal Dynamics and Chaos in Semiconductors (Cambridge University Press, Cambridge, 2001).

[7] F. Qin, E. E. Wolf, and H.-C. Chang, Phys. Rev. Lett. 72, 1459 (1994).

[8] I. Aranson, H. Levine, and L. Tsimring, Phys. Rev. Lett. 72, 2561 (1994).

[9] G. A. Johnson, M. Löcher, and E. R. Hunt, Phys. Rev. E 51, R1625 (1995).

[10] A. Hagberg, E. Meron, I. Rubinstein, and B. Zaltzman, Phys.
Rev. Lett. 76, 427 (1996).

[11] R. Martin, A. J. Scroggie, G.-L. Oppo, and W. J. Firth, Phys. Rev. Lett. 77, 4007 (1996).

[12] Y. Tabe and H. Yokayama, Langmuir 11, 4609 (1995).

[13] A. S. Mikhailov and K. Showalter, Phys. Rep. 425, 79 (2006).

[14] V. N. Biktashev and A. Holden, J. Theor. Biol. 169, 101 (1994).

[15] V. S. Zykov, G. Bordiougov, H. Brandtstädter, I. Gerdes, and H. Engel, Phys. Rev. E 68, 016214 (2003).

[16] W. Vance and J. Ross, J. Chem. Phys. 103, 2472 (1995).

[17] L. Glass and W. Zh. Zeng, Ann. N.Y. Acad. Sci. 591, 316 (1990).

[18] C. Utzny, W. Zimmermann, and M. Bär, Europhys. Lett. 57, 113 (2002).

[19] S. Rüdiger, D. G. Miguez, A. P. Munuzuri, F. Sagués, and J. Casademunt, Phys. Rev. Lett. 90, 128301 (2003).

[20] M. Henriot, J. Burguete, and R. Ribotta, Phys. Rev. Lett. 91, 104501 (2003).

[21] D. G. Miguez, E. M. Nicola, A. P. Munuzuri, J. Casademunt, F. Sagués, and L. Kramer, Phys. Rev. Lett. 93, 048303 (2004).

[22] S. Zykov, V. S. Zykov, and V. Davydov, Europhys. Lett. 73, 
EXTERNAL FORCING AND FEEDBACK CONTROL OF...

PHYSICAL REVIEW E 75, 066203 (2007)

335 (2006).

[23] N. J. Wu, H. Zhang, H.-P. Ying, Z. Cao, and G. Hu, Phys. Rev. E 73, 060901(R) (2006).

[24] T. Okuzono and T. Ohta, Phys. Rev. E 64, 045201(R) (2001).

[25] T. Okuzono and T. Ohta, Phys. Rev. E 67, 056211 (2003).

[26] S. Sugiura, T. Okuzono, and T. Ohta, Phys. Rev. E 66, 066216 (2002).
[27] T. Ohta and H. Tokuda, Phys. Rev. E 72, 046216 (2005).

[28] H. Tokuda and T. Ohta, J. Phys. Soc. Jpn. 75, 064005 (2006).

[29] A. M. Turing, Philos. Trans. R. Soc. London, Ser. B 237, 37 (1952).

[30] A. Pikovsky, M. Rosenblum, and J. Kurths, Synchronization: A Universal Concept in Nonlinear Sciences (Cambridge University Press, Cambridge, 2001). 